ISSN electrónico: 2172-9077

https://doi.org/10.14201/fjc201816135154

\title{
COMUNICACIÓN ORAL Y M-LEARNING EN EL AULA DE INGLÉS DE PRIMARIA: LA FOTOGRAFÍA Y EL VÍDEO COMO RECURSO
}

\section{Oral Communication and M-Learning in the Primary English Classrooms: Photography and Video as a Resource}

\author{
Marta GARCÍA-SAMPEDRO \\ Profesora Asociada. Universidad de Oviedo, España \\ E-Mail: garciafmarta@uniovi.es \\ (D) http://orcid.org/0000-0003-1523-1314
}

Mirian MIRANDA MORAIS

Investigadora predoctoral. Universidad de Oviedo, España

E-Mail: mirian.mirandamorais@,cpraviles.es

(i) http://orcid.org/0000-0002-8375-4371

\section{Eva M. ${ }^{a}$ INESTA MENA}

Profesora. Universidad de Oviedo, España

E-Mail: inestaeva@,uniovi.es

(iD http://orcid.org/0000-0001-6453-2776

\begin{abstract}
RESUMEN
En este artículo se presenta el proceso de diseño, implementación y evaluación del proyecto 'Films \& Photographs: An M-learning Experience in Primary Education' que se desarrolló a través la metodología propia de la investigación-acción. El objetivo fundamental del proyecto era fomentar la comunicación oral en lengua inglesa (como lengua extranjera) utilizando los dispositivos móviles en la Educación Primaria a través del uso de la fotografía y el video. En el proyecto participaron dos docentes de lengua inglesa de primaria, otros dos del ámbito universitario y una asesora del Centro de Profesorado y Recursos. El alumnado participante, de entre diez y doce años, pertenecía a dos centros escolares del Principado de Asturias, España y el proyecto fue desarrollado durante el curso 2015-2016. Las técnicas de recogida de información utilizadas fueron la observación participante, la entrevista semi-estructurada y el cuestionario. Las actividades se centraron en utilizar la tecnología móvil (smartphones y tablets) para la producción y edición de videos y fotografías. Los resultados muestran la percepción positiva del profesorado y del alumnado participante sobre el m-learning pues consideran que ha influido directamente en la motivación hacia la realización de las tareas propuestas. Todo ello repercute en la mejora de la competencia comunicativa, tecnológica y artística. Se trata, por tanto, de una propuesta novedosa que conjuga la integración de aprendizajes lingüísticos, tecnológicos y artísticos en el contexto de la Educación Primaria.
\end{abstract}

Palabras clave: Comunicación oral; m-learning; enseñanza lengua inglesa; escuela primaria.

\section{ABSTRACT}

The process of design, implementation and evaluation of the project 'Films \& Photographs: An M-learning Experience in Primary Education' is presented in this article through the research-action methodology. The project's main objective was to foster oral communication in the English language (as an additional language) using mobile 


\begin{abstract}
devices in primary education through photography and video. Two English primary language teachers, two university lecturers and an in-service teacher advisor from the Teachers' Training and Resource Centre participated in the project. The pupils, around ten and twelve years of age, belonged to two different schools in The Principality of Asturias, Spain and the project was developed during the school year 2015-2016. The techniques used to collect data were participant observation, semi structured-interviews and a questionnaire. The activities were focused on the use of mobile devices (smartphones and tablets) to produce and edit videos and photographs. The results show students and teachers' positive perception about m-learning since they consider it has directly influenced the motivation to carry out the proposed tasks. All this has an effect on the improvement of the communicative, technological and artistic competence in the primary education context.
\end{abstract}

Key words: Oral communication; m-learning; English language teaching; Primary Education.

\title{
1. INTRODUCCIÓN
}

En nuestro cerebro, lo emocional y lo cognitivo son distinguibles, pero no separables y por ello, desde una perspectiva neuronal, lo afectivo es parte integral de lo cognitivo (Schumann, 1994). El contexto contemporáneo para el aprendizaje-enseñanza de las lenguas es muy complejo y requiere que el profesor integre con éxito todos los elementos necesarios de un modo efectivo, incluida la motivación. Por ello, la introducción de nuevas tecnologías en el aula junto con los materiales apropiados y una pedagogía adaptada a las necesidades del alumnado contribuirá a abordar el desafío que supone la enseñanza de lenguas en nuestros días (Levy, 2012).

Educar es comunicar; ambas acciones tienen muchos rasgos en común pues implican determinados procesos cognoscitivos relacionados con el manejo y transmisión de la información y comparten métodos, técnicas y recursos (Martínez-Salanova, 2016). El éxito en la comunicación supone una comprensión significativa de la información, lo cual repercute en la construcción de conocimientos, y en que estos se tornen más complejos y elaborados. En los años 70 y 80 del siglo XX surge la educomunicación como un nuevo campo de estudios teórico-práctico que conecta la educación y la comunicación. Esta disciplina, que nace con Freinet, se convierte en un campo de estudios heterogéneo y plural que continúa expandiéndose con las aportaciones de Freire, Kaplún o McLaren entre otros (Barbas, 2012). Se puede decir, por tanto, que la competencia comunicativa es una competencia clave para el aprendizaje que debemos tener en cuenta en todas y cada una de las propuestas educativas que se programan (Alsina, Cañabate y de la Creu, 2013).

Para ser competentes en comunicación es necesario desarrollar todas las destrezas lingüísticas (Hymes, 1972), sin embargo, la enseñanza de la lengua oral ha estado absolutamente relegada en la mayoría de las escuelas en España y este abandono, casi generalizado, ha tenido repercusiones muy negativas en la enseñanza oral de segundas lenguas. Podría decirse que la enseñanza de la lengua oral no forma parte de nuestra tradición escolar, lo que explicaría en cierto modo las carencias actuales en este campo. En la segunda mitad del s. XX la lengua oral se integró en los primeros cursos escolares, pero rápidamente la atención se dirigía al aprendizaje de la lectoescritura, asumiendo que las habilidades orales ya se desarrollaban en al ámbito familiar y social (Vilá y Castellá, 2014; 2015).

Estos autores destacan el papel central de la interacción oral para vertebrar la vida diaria del aula y la complejidad que presenta la enseñanza de la oralidad, que no suele percibirse, ni en el contexto de la lengua materna, ni en el de otras lenguas (Goh \& Burns, 2012). En este sentido, Ballesteros y Palou (2005) opinan que a la mayor parte del profesorado le resulta poco apetecible la enseñanza de las destrezas orales, pues consideran difícil su enfoque, organización y evaluación. Vilá y Castellá (2015) señalan que las clases de lengua han de ser vivenciales y coinciden con Kaplún (1998) cuando dice que «se aprende de verdad lo que se vive, lo que se recrea, lo que se reinventa y no lo que simplemente se lee y se escucha» (p. 51). 
En cuanto a la enseñanza de la comunicación oral en segundas lenguas o lenguas extranjeras se diría que la necesidad de dominar las destrezas orales está más que asumida por todos; no obstante, a tenor de los resultados de las evaluaciones nacionales e internacionales que mencionaremos más adelante, tiene sentido cuestionar las metodologías aplicadas.

Esta búsqueda de nuevos enfoques educativos para mejorar la enseñanza de la lengua oral es la que hace incorporar el m-learning al aula de lengua inglesa de primaria. Fombona y Pascual (2013, p. 210) describen el aprendizaje con dispositivos móviles, mobile learning o m-learning como «el uso de pequeños equipos portátiles, principalmente smartphones y tablets en educación» y añaden que «estos aparatos permiten una gestión informática de los datos y conectividad inalámbrica para la interacción telemática en el proceso de enseñanza-aprendizaje». Se puede decir que el m-learning se orienta hacia un aprendizaje colaborativo, flexible, espontáneo e informal basado en la resolución de problemas. Estas características han favorecido su rápida incorporación en las prácticas educativas y «han redibujado el panorama educativo, aportando a la educación no sólo movilidad sino también conectividad, ubicuidad y permanencia» (Cantillo, Roura y Sánchez, 2012, p. 3).

Además de todas estas características, lo que sin duda contribuye al éxito del m-learning es la motivación que despierta en el alumnado. No se debe olvidar que la motivación es uno de los campos de estudio principales en el aprendizaje de lenguas desde hace varias décadas y desde entonces se reconoce como una característica diferenciadora que nos puede ayudar a entender por qué algunas personas tienen más éxito que otras a la hora de aprender una lengua (Dörnyei, 2001; 2002; 2005) y tal como apunta Scovel, (2000), la motivación, la afectividad y las emociones influyen considerablemente en el aprendizaje de lenguas y por ello es fundamental cuidar el clima emocional del aula (Ibarrola, 2013).

Hoy en día, el teléfono móvil ha pasado de ser un objeto tecnológico a ser un objeto cultural del que se ha apropiado toda la sociedad y que ha trascendido más allá del ámbito puramente comunicativo (López y Gomez, 2016). Estos autores, coincidiendo con Gardner y Davis (2014), consideran que la aparición del teléfono móvil ha supuesto un cambio social que lleva aparejados múltiples cambios en todos los ámbitos. Fombona y Pascual (2013) opinan que «los dispositivos móviles son un nuevo soporte de los medios y suponen un desafío a los planteamientos educativos en una triple dimensión espacial, temporal y social» (2013, p. 211). En el contexto educativo español, el uso del teléfono móvil y las tablets va emergiendo tímidamente. Por ejemplo, en algunos centros educativos de nuestro país, privados ${ }^{1}$ en su mayoría, comienzan a utilizarse tablets que sustituyen a los manuales escolares y son una herramienta de trabajo muy completa. Sin embargo, no todos los centros permiten la utilización de dispositivos móviles, aunque solo sea para un uso puntual en proyectos con fines educativos. Existen todavía muchas restricciones en cuanto a su uso. En este sentido, Cantillo et al. (2012) opinan que la escuela se distancia, una vez más, de la realidad vivida por el alumnado. En cualquier caso, son muchas las ventajas que aporta el m-learning, tal como explican estos autores: el tamaño reducido de los dispositivos; la inmediatez y conectividad a redes inalámbricas; la eliminación de barreras espaciales o temporales en el aprendizaje; la adaptabilidad de servicios, aplicaciones e interfaces a las necesidades del usuario y, por último, la posibilidad de incluir accesorios tales como teclados o lápices que facilitan su uso.

Las últimas leyes educativas (LOE, 2006 y LOMCE, 2013) consideran las destrezas orales como fundamentales, por lo que el interés por la enseñanza de las mismas ha aumentado considerablemente.

\footnotetext{
${ }^{1}$ En la investigación que los autores de este artículo están realizando en la actualidad, se constata como algunos centros privados del Norte de España (Compañía de Jesús, Alter Vía), al igual que otros en Madrid (San Patricio, Casvi, Mirabal) o en Barcelona (Montserrat, Garbí-Pere Vergés) utilizan las tablets o i-pads en sustitución de los libros de texto.
}

Ediciones Universidad de Salamanca / CC BY - NC ND $\quad$ Fonseca, Journal of Communication, n. 16, 2016, pp. 135-154 
Sin embargo, no se constata una coherencia entre las necesidades de la sociedad, el mundo laboral y la enseñanza real si tenemos en cuenta los poco alentadores resultados obtenidos por los alumnos españoles tanto en las 'Evaluaciones de Diagnóstico' como en los informes europeos consultados: 'Estudio Europeo de Competencia Lingüística' (2012) y 'The assessment of pupil' skills in English in eight countries' (2002). Por ello, siguen siendo necesarias propuestas didácticas innovadoras que repercutan en una mejora del aprendizaje, así como su divulgación. Precisamente, esa inquietud por buscar nuevas propuestas y estrategias de enseñanza novedosas motiva el presente artículo, cuya finalidad es presentar el proceso de diseño, implementación y evaluación de un proyecto que integra una investigación-acción colaborativa entre docentes de centros educativos y la universidad con el objetivo de mejorar la competencia comunicativa en inglés del alumnado de primaria y promover la reflexión sobre la incorporación de las tecnologías móviles en la educación y su importancia en el aula de lenguas extranjeras. Conociendo la incidencia positiva del uso del m-learning en la práctica docente, se estimó oportuno desarrollar un proyecto educativo que contribuyese a la innovación metodológica en estas áreas. De este modo, surge el proyecto 'Films \& Photographs: An M-learning Experience in Primary Education' que pretende contribuir a la innovación metodológica para la enseñanza de la comunicación oral en inglés, incorporando el uso de dispositivos móviles. El proyecto puesto en marcha en el curso 2015-2016 al amparo del convenio de colaboración entre la Consejería de Educación y Ciencia del Principado de Asturias y la Universidad de Oviedo en la Convocatoria de Proyectos de Innovación e Investigación para la realización de actividades educativas. El interés fundamental del proyecto se centra en mejorar la competencia comunicativa del alumnado en lengua inglesa, desarrollar su competencia digital de una forma crítica, responsable y segura y fomentar la competencia artística del alumnado y su creatividad.

\section{OBJETIVOS}

El objetivo general de este artículo es presentar el proceso de diseño, implementación y evaluación del proyecto 'Films \& Photographs: An M-learning Experience in Primary Education', utilizando la metodología propia de la investigación-acción. Además, tiene unos objetivos específicos como son:

-Identificar propuestas constatadas de enseñanza-aprendizaje de m-learning y de promoción de la lengua oral en lengua extranjera.

-Analizar la percepción del profesorado y del alumnado sobre la implementación y desarrollo del proyecto.

-Contrastar la relevancia del uso m-learning en la mejora de la motivación del alumnado.

\section{METOdOLOGÍA Y TÉCNICAS EMPLEADAS}

En base a los objetivos planteados se presenta el proceso de diseño, aplicación y evaluación de este proyecto de innovación para la incorporación de las tecnologías móviles en el aula de lengua inglesa utilizando una metodología basada en la investigación-acción. Esta se caracteriza por el papel activo que asume el profesorado que forma parte de la investigación, el cual parte de los problemas que se encuentra en su práctica educativa, reflexiona sobre los mismos e intentan comprenderlos y mejorarlos. El precursor de esta metodología fue Lewin (1946), a quien siguieron otros autores como Elliot (1990) o Stenhouse (1975), más centrado en el ámbito educativo. Atendiendo a la clasificación de Reason (1994) citada en Rodríguez, Gil y García (1996, p. 53) esta investigación-acción se enmarca en la modalidad de investigación cooperativa al caracterizase por la decisión de profesionales de más de dos ámbitos, unos ligados al campo profesional y otros pertenecientes al ámbito académico, de 
agruparse para resolver juntos problemas que atañen a sus prácticas docentes. Al planificar la secuencia del estudio se tuvieron en cuenta las consideraciones de la línea de investigación-acción denominada Lesson Study. Esta se caracteriza por ser una investigación-acción colaborativa que realizan un grupo reducido de docentes para diseñar, desarrollar, comprobar, criticar y mejorar una propuesta didáctica (lesson) y de la cual se concretan los aprendizajes claves, se analiza a cada docente y se escucha al alumnado (Soto y Pérez-Gómez, 2015). Para establecer las fases del estudio se tuvo en cuenta la secuencia de pasos de la propuesta clásica de Lewin (1946): planificación, acción, observación y reflexión, y tomando en cuenta estas premisas y el contexto del estudio que se presenta, se diseñó una secuencia de cinco fases (añadiendo una fase preliminar) que aparece recogida en la Figura 1:

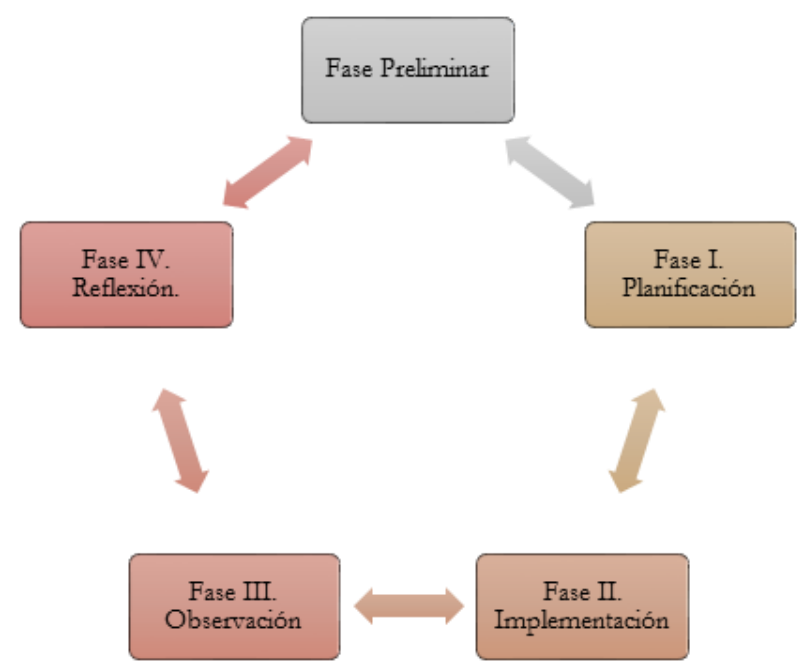

Figura 1. Fases y ciclos de la investigación-acción

Para recoger información de las distintas fases de la investigación se optó por combinar diversas técnicas de recogida y análisis de datos de naturaleza cuantitativa y cualitativa. Estas son la observación participante, la entrevista y el cuestionario.

\subsection{Participantes}

El grupo de investigación-acción en el marco del proyecto 'Films \& Photographs: An M-learning Experience in Primary Education' está compuesto por dos profesoras de lengua inglesa que imparten enseñanzas en Educación Primaria, dos profesoras del Departamento de Ciencias de la Educación y una asesora del Centro de Profesores y Recursos que ha colaborado en cuestiones relativas al proceso de investigación. Las profesoras que imparten docencia en los dos centros educativos de primaria que han participado en el proyecto cuentan con una dilatada experiencia docente en cursos con alumnos entre 10 y 12 años, han participado en proyectos de innovación en otras ocasiones y tienen una competencia digital media, media-alta. La muestra de alumnado que participó del estudio fue de 101 alumnos que cursaban los niveles de $5 .^{\circ}$ y $6 .^{\circ}$ de Educación Primaria y que pertenecían a dos centros educativos distintos, uno público y otro privado sostenidos con fondos públicos, situados en la zona 
centro de la ciudad y con un estrato social medio-bajo. En ambos centros nos encontramos con alumnado diverso, contando con alumnado de necesidades específicas de apoyo educativo y un porcentaje elevado de alumnado de diferentes países.

\subsection{INSTRUMENTOS}

Siguiendo la secuencia de la investigación-acción se optó por recoger datos de distinta naturaleza a través de diferentes agentes (profesorado y alumnado) utilizando a lo largo del proceso de investigación las siguientes técnicas de recogida de información e instrumentos:

- Diario de investigación. Se utilizó a lo largo del proceso de planificación y desarrollo de la investigación y de la implementación del proyecto de innovación en el aula. Esta tarea la llevaron a cabo las profesoras que implementaron el proyecto. En el diario de investigación se recogía información sobre la planificación del proyecto, los acuerdos adoptados por el equipo investigador en las reuniones de planificación e información relevante sobre la implementación de las sesiones, el desarrollo de las mismas y la evaluación.

- Notas de campo. Se utilizaron para recoger información relevante y apreciaciones personales en el proceso de implementación del proyecto.

- Cuestionario elaborado ad hoc dirigido al alumnado. Se utilizó al final del proyecto con objeto de obtener información sobre la opinión y la percepción del alumnado participante sobre la experiencia en los ámbitos de comunicación oral en lengua inglesa, competencia tecnológica, artística y motivación. El cuestionario que se aplicó es de elaboración propia denominado 'Cuestionario de valoración uso de dispositivos móviles en el aula de inglés'. Lo componen preguntas abiertas, cerradas y preguntas de elección múltiple. El tipo de variables y el tipo de ítems que se recogen en dicho instrumento se presentan en la Tabla 1.

Tabla 1. Tipo de información recogida y tipo de preguntas

\begin{tabular}{|c|c|}
\hline Tipo de información & Tipo de preguntas \\
\hline Información sociodemográfica. & Ítems de construcción e ítems de elección. \\
\hline $\begin{array}{c}\text { Opiniones sobre competencia comunicativa, } \\
\text {-learning, competencia artística y competencia } \\
\text { tecnológica. }\end{array}$ & $\begin{array}{c}\text { Escala Likert (12 ítems) 4 opciones: 1 (Muy en } \\
\text { Desacuerdo) hasta el 4 (Muy de Acuerdo). }\end{array}$ \\
\hline $\begin{array}{c}\text { Opiniones sobre aspectos que favorecen la } \\
\text { motivación. }\end{array}$ & Ítems de construcción. \\
\hline
\end{tabular}

Fuente: Elaboración propia.

Los cuestionarios se aplicaron a través de un formulario online en el aula de informática del centro. Se informó a la dirección del centro, las familias y al alumnado participante de la confidencialidad y anonimato de los datos obtenidos y de su posible utilización en el marco de la difusión de la investigación.

- Entrevista semi-estructurada ad-hoc dirigida al profesorado. Con objeto de recoger información acerca de la percepción de las profesoras participantes en el proyecto se diseña una entrevista semi-estructurada. Esta tiene por objeto recoger información sobre la valoración y evaluación que las profesoras realizan del proyecto. La entrevista se realizó a las 
cuatro colaboradoras en el proyecto. Para diseñar las preguntas que conforman la entrevista semi-estructurada tomando como referencia la bibliografía consultada y la finalidad del proyecto se definieron las siguientes categorías: innovación, competencia digital, competencia artística, competencia comunicativa, cooperación docente, formación del profesorado y motivación. En la Tabla 2 recogemos los principales descriptores para cada una de las categorías planteadas en la entrevista.

Tabla 2. Descriptores de las categorías planteadas en el diseño de la entrevista semi-estructurada

\begin{tabular}{|l|l|}
\hline \multicolumn{1}{|c|}{ Categorías } & \multicolumn{1}{c|}{ Descriptores } \\
\hline Innovación & $\begin{array}{l}\text { Participación en proyectos. } \\
\text { Utilización de metodologías que movilicen pensamiento y acción. }\end{array}$ \\
\hline $\begin{array}{l}\text { Competencia } \\
\text { digital }\end{array}$ & $\begin{array}{l}\text { Uso de dispositivos móviles. } \\
\text { Aplicaciones y uso del móvil. }\end{array}$ \\
\hline $\begin{array}{l}\text { Competencia } \\
\text { artística }\end{array}$ & Producción de actividades artísticas. \\
\hline $\begin{array}{l}\text { Competencia } \\
\text { comunicativa }\end{array}$ & Producción de textos orales. \\
\hline $\begin{array}{l}\text { Cooperación } \\
\text { docente }\end{array}$ & $\begin{array}{l}\text { Trabajo en equipo, reparto de tareas, roles y responsabilidades. } \\
\text { Espacios y tiempos de coordinación. }\end{array}$ \\
\hline $\begin{array}{l}\text { Formación del } \\
\text { profesorado y } \\
\text { motivación }\end{array}$ & $\begin{array}{l}\text { Asistencia a cursos de formación. } \\
\text { Autoformación. } \\
\text { Estrategias motivacionales. }\end{array}$ \\
\hline
\end{tabular}

Fuente: Elaboración propia.

\subsection{Procedimiento}

Como se ha explicado anteriormente, esta investigación-acción utiliza la secuencia clásica de Lewin (1946) a la que se ha añadido una Fase preliminar. A continuación se muestra el cronograma del trabajo desarrollado:

- Primer trimestre del curso: Elaboración y presentación de la propuesta de proyecto a la Consejería de Educación. Revisión de bibliografía para fundamentar el proyecto.

- Segundo trimestre: Diseño y planificación del proyecto.

- Tercer trimestre: Implementación del proyecto en el aula, recogida de datos y análisis de datos; elaboración del informe de investigación.

\section{Fase preliminar.}

Este proyecto de innovación surge en el marco de la Convocatoria de Proyectos de Innovación de la Consejería entre centros educativos y la Universidad. En este primer momento el profesorado identifica algunas de las necesidades que se plantean en las aulas de inglés de primaria como son la escasez de actividades de comunicación oral que llevan por tanto a una escasa fluidez a la hora de expresarse en lengua inglesa. En muchos casos, la metodología es poco atractiva, por lo que se entiende que la mejora de las estrategias metodológicas y motivacionales del profesorado ayudarán a mejorar la competencia oral en inglés y a la motivación para participar en las actividades de interacción.

Siguiendo las premisas planteadas anteriormente, el profesorado implicado planificó las actividades y planteó posibles mejoras en la metodología de enseñanza; se estudiaron propuestas teóricas y experiencias sobre $m$-learning y se decidió adoptar un enfoque interdisciplinar para fomentar 
competencias comunicativas, tecnológicas y artísticas, mejorar la motivación del alumnado y la responsabilidad en el uso de la tecnología móvil.

Durante esta fase preliminar, que se llevó a cabo durante el primer trimestre del curso 2015-16, se llevaron a cabo reuniones del equipo de investigador, la primera se dedicó a esbozar el planteamiento del problema, diseño de la investigación y planteamiento del proyecto a desarrollar. El resto de sesiones se abordó la revisión de investigaciones, bibliografía y prácticas.

Fase I. Planificación.

Finalizada la fase preliminar, el equipo investigador mantuvo el procedimiento de trabajo a través de reuniones e intercambio de datos a través del correo electrónico. En esta ocasión, el trabajo se centró en la elaboración y planificación de la propuesta didáctica, y en el diseño y el procedimiento de recogida de datos a lo largo de la investigación.

El profesorado identificó los objetivos y contenidos curriculares que podrían trabajarse; eligió los dispositivos móviles y las aplicaciones pertinentes; se presentó el proyecto contextualizado al alumnado y se negoció con ellos acerca de distintos aspectos estructurales y metodológicos (gestión de grupos, tiempos de aplicación).

Descripción del proyecto de Innovación 'Films \& Photographs: An M-learning Experience in Primary Education'.

En este proyecto confluyen las TIC (tecnologías de la información y la comunicación) y las TAC (Tecnologías del aprendizaje y el conocimiento en torno al m-learning). Los dispositivos se emplean para la producción de imágenes, fotografías o vídeos integrando estos recursos en las actividades de lengua para mejorar la destreza comunicativa del alumnado en inglés dando prioridad a la oralidad (hablar, conversar y escuchar). Esto posibilita un enfoque interdisciplinar que contribuye al desarrollo de la competencia en comunicación oral, junto con las competencias tecnológicas y artísticas.

El proyecto 'Films \& Photographs: An M-learning Experience in Primary Education' tiene los siguientes objetivos:

- Potenciar la comunicación oral en lengua inglesa a través del uso de dispositivos móviles.

- Fomentar el aprendizaje a través de dispositivos móviles (m-learning).

- Mejorar la motivación del alumnado.

- Contribuir al desarrollo de las competencias tecnológicas y artísticas del alumnado desde el área lingüística.

El proyecto se caracteriza por plantear actividades lingüísticas y actividades en las que se han de usar herramientas tecnológicas, concretamente aplicaciones móviles de vídeo y fotografía, para lo que se desarrollan dos tipologías de actividades diferenciadas: actividades de fotografía y actividades de vídeo. Destacar que tanto en las actividades fotográficas como en las de video se propone un tema y en ningún caso se permiten imágenes o videos en los que aparezca el alumnado. Las temáticas son muy variadas, dependiendo de los contenidos que en ese momento se estén trabajando en el aula de inglés y en el caso de los videos los alumnos han de crear sus propios personajes utilizando cualquier material o recurso: muñecos, marionetas u otros.

Actividades de fotografía:

En el caso de la realización de actividades con fotografías la temporalización estimada es de tres sesiones, en función del número de estudiantes del aula. El alumnado saca las fotografías y las edita durante el fin de semana. Luego las envía a la profesora y en el aula se realizan las actividades de descripción y comentario de las mismas desde la perspectiva del diálogo creativo. Las fotografías se toman habitualmente con el móvil de los alumnos o con el de sus padres o familiares. 
Para las actividades fotográficas se han utilizado las aplicaciones Snapseed y PicsArt. y se han realizado las siguientes actividades:

- Toma de fotografías.

- Edición.

- Montaje (opcional).

Las actividades lingüísticas desarrolladas a partir de las fotografías realizadas incluyen:

- Descripción: Colores, texturas, formas, objetos, personas, paisajes, animales, expresión de sentimientos, gustos, etc.

- Entrevista.

- Invención de una historia.

- Elaboración de un anuncio publicitario.

- Debate.

- Diálogo.

Actividades de vídeo.

La temporalización de las actividades de vídeo es de unas cuatro semanas, aproximadamente un total de 15 sesiones. Esto varía en función del número de alumnado del aula. La primera semana se suele dedicar al diseño del texto o guion, la segunda a la elección, fabricación o transformación de materiales para elaboración de los personajes (marionetas, juguetes, etc.). En la tercera semana se suelen preparar los decorados y en la cuarta semana se realiza la grabación.

En cuanto a las aplicaciones previstas para la edición y montaje de vídeo en dispositivos móviles se presentan para Android: VidTrim, We Video y Viva Video y para Apple: Imovie y Vimeo.

La secuencia de actividades es la siguiente:

Fases para la elaboración de un video:

- Desarrollo de la idea: crear un argumento, comienzo-desarrollo-desenlace. Elaborar una breve sinopsis de la historia, entrevista, anuncio, etc.

- Elaboración del guion técnico, en el que quedarán reflejados datos como la escena, el tipo de plano, la localización, el momento del día/noche, voz, sonido y los efectos que se quieran añadir.

- Toma de fotografías (opcional).

- Edición (opcional).

- Montaje.

Las actividades lingüísticas que se realizan paralelas a la elaboración de los videos son:

- Elaboración del guion.

- Grabación de presentaciones, diálogos, descripciones o textos.

- Redacción de los créditos.

En el transcurso de esta fase el equipo investigador planifica el procedimiento de recogida de información a lo largo del proceso. Estos aspectos aparecen recogidos en el apartado instrumentos.

Fase II. Implementación.

Se informa a los equipos directivos de los centros, al resto de profesorado de Lengua Inglesa y al alumnado y sus familias de las condiciones de participación en este proyecto. De manera explícita se comunica que los datos obtenidos podrían ser difundidos en el campo de la investigación y se garantiza el anonimato del alumnado participante. Diseñado el proyecto, las dos profesoras de los centros pasan a desarrollar el proyecto en el aula. Este proceso se inicia con una sesión de trabajo cooperativo entre las profesoras y el alumnado participante. Se dedica una sesión a presentarlo 
contextualizado y negociando los distintos aspectos estructurales y metodológicos (gestión de grupos, tiempos de aplicación). Seguidamente se pasa a desarrollar el proyecto en base a la secuencia descrita en la Fase de Planificación, concretamente en el apartado referido a la Descripción del Proyecto. El proceso de implementación tiene una duración de 9 semanas. Una vez finalizado el proyecto en el aula el alumnado comparte las tareas finales del trabajo en grupos (producto) y se difunde a través de un blog.

Fase III. Observación.

De manera paralela a la Fase de Implementación se realiza una recogida de información sistemática de lo acontecido en las sesiones a través de la observación participante con la ayuda de los instrumentos creados a tal efecto (diarios y notas de campo). Se crean instrumentos de evaluación para realizar el seguimiento de las actividades y productos desarrollados por el alumnado en el aula como son el portfolio digital y las rúbricas, pues facilitan la valoración del trabajo y la toma de decisiones durante el proceso. Tanto la recogida de información como la reflexión acerca de los datos se tienen en cuenta para la toma de decisiones para planificar y realizar ajustes en el proceso de enseñanzaaprendizaje. Finalizado el proceso de implementación se recogen y ordenan todos los datos obtenidos para poder realizar su análisis posterior. Los datos se organizan en función de su naturaleza y procedencia atendiendo a las categorías diseñadas y a continuación se procede al análisis de los mismos y se organizan los resultados.

Fase IV. Reflexión.

En base a los resultados obtenidos, el equipo de investigación se reúne y comparte sus impresiones y reflexiones sobre la puesta en marcha del proyecto, así como los aspectos que se deben modificar y mejorar. Después, se elabora un informe en el que se recogen los puntos débiles, fuertes y aquellos aspectos que deben ser mejorados en cursos posteriores.

\subsection{ANÁLISIS DE DATOS}

Para el análisis de resultados se contempló el uso de análisis de datos de carácter cuantitativo y cualitativo al manejar resultados de distinta naturaleza. Los datos cuantitativos que se obtuvieron a través de los cuestionarios y fueron procesados a través del paquete estadístico SPSS (versión 21) para el análisis de las opiniones del alumnado obtenidas a través de la escala Likert, que se concreta en el uso de la estadística descriptiva.

Para el análisis de la información cualitativa recogida a través del diario de investigación, el trabajo de campo, las preguntas abiertas del cuestionario y la entrevista semi-estructurada se utilizó el análisis de contenido tomando como referencia la propuesta de Rodríguez, Gil y García (1996). Estos autores consideran que, en el análisis cualitativo de datos, el tratamiento que se lleve a cabo debe preservar su naturaleza textual, poniendo en práctica tareas de categorización. A la hora de organizar esta información se tomaron como referencia, en el caso de las notas de campo y diario de investigación, las siguientes categorías: competencia comunicativa, competencia tecnológica, competencia artística y motivación. Estas tienen su origen en la revisión bibliográfica realizada en el marco de planificación del proyecto y responden a la finalidad del mismo.

En el caso de la entrevista semi-estructurada se utilizaron las mismas categorías planteadas por el equipo investigador en el proceso de diseño del instrumento. Para completar el análisis de los datos cualitativos se aportaron citas textuales significativas que ejemplifican las opiniones recogidas mediante las entrevistas. 


\section{RESUltados}

Los resultados obtenidos se presentan en tres bloques diferenciados: resultados obtenidos a través de la observación participante (notas de campo y diario de investigación), resultados obtenidos a través del cuestionario dirigido al alumnado y resultados de las entrevistas semi-estructuradas dirigidas a las profesoras participantes en el proyecto.

\subsection{OBSERVACIÓN PARTICIPANTE}

Las categorías utilizadas para analizar los datos obtenidos a través de las notas de campo y del diario de investigación fueron: competencia comunicativa, competencia tecnológica, competencia artística y motivación.

En relación a la competencia comunicativa se puede decir que los alumnos, en general, hicieron un gran esfuerzo por comunicarse en lengua inglesa. En las actividades con fotografías, los alumnos se esforzaron por explicar dónde, cómo y cuándo tomaron las fotografías y qué aplicaciones utilizaron para editarlas. En general, se sintieron muy orgullosos de sus productos y estaban deseosos de hablar sobre ellos.

En el caso de las actividades de video, los alumnos se esforzaron por mejorar la pronunciación y la entonación de sus textos orales, puesto que la actividad ha de ser grabada y difundida en el centro escolar. En cuanto a la competencia artística se puso de manifiesto el interés por mejorar los aspectos creativos, por la búsqueda de la originalidad de los productos y por experimentar con las distintas aplicaciones utilizadas. En las actividades de video ocurrió lo mismo, destacando el interés del alumnado por mejorar su creatividad utilizando materiales diferentes, diseñando decorados acordes con la estética de la historia o textos alternativos, como pueden ser variaciones de cuentos tradicionales, anuncios televisivos, etc. La competencia tecnológica del alumnado se desarrolló indudablemente gracias al uso de nuevas aplicaciones, así como la responsabilidad de uso de los dispositivos móviles, el conocimiento sobre la versatilidad de los mismos y sus usos educativos. Por último, se prestó atención a la motivación del alumnado, que indudablemente mejoró mucho, no solo por el uso de las tecnologías móviles, sino también por la libertad que tuvieron para crear sus productos artísticos, emplear técnicas artísticas y materiales alternativos y sobre todo por abandonar las tediosas rutinas escolares y el uso tóxico que de las tecnologías se hace en la escuela cuando los escolares son sujetos pasivos de las mismas y no se produce ningún tipo de creación (Acaso, 2013).

\subsection{Cuestionario}

Del total del alumnado que participó en el proyecto $(\mathrm{N}=101)$ respondieron al cuestionario un $84 \%$ $(\mathrm{N}=84)$. El alumnado contaba con edades comprendidas entre los 10 y los 12 años. Un 38,1\% eran niños y un $61,9 \%$ niñas. Un $38,1 \%$ de $6^{\circ}$ curso y un $61,9 \%$ se encontraba cursando $5^{\circ}$ curso de Educación Primaria.

En relación a la competencia comunicativa los resultados ponen de manifiesto que la mayoría del alumnado opina que el uso del teléfono móvil les ha ayudado a mejorar su comunicación oral y su comprensión del inglés, como se recoge en el Gráfico1. 
Gráfico 1. Opinión del alumnado sobre lo que les ha ayudado a mejorar su comunicación y comprensión en inglés

\section{E1 uso de los dispositivos móviles en el aula de inglés me han ayudado en mejorar en:}

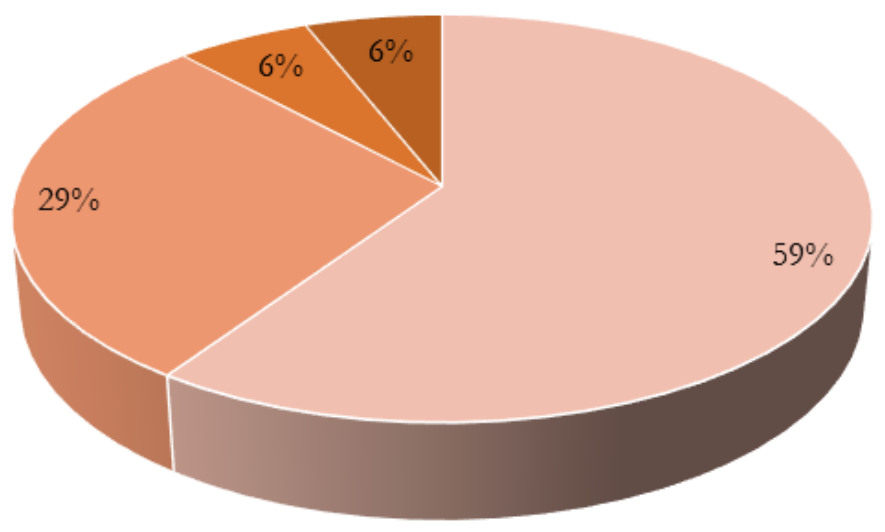

$=$ Hablar

- Comprender

- Escribir

- Leer

Fuente: elaboración propia.

Si añadimos nuevas informaciones procedentes del cuestionario, advertimos, como aparece recogido en el Gráfico 2, que el alumnado considera que las actividades del proyecto han ayudado a mejorar su comunicación en lengua inglesa, cabe señalar que solamente 6 alumnos/as, consideran la opción «poco», en contraste con las puntuaciones que otorgan la mayoría del alumnado que oscilan entre «bastante» $(39,3 \%$ ) y «mucho» (53,6 \%), ver el Gráfico 2. Siendo la actividad de grabación y edición de video con tablet o móvil la más valorada por todo el alumnado. En este gráfico se proponen solamente los datos de respuesta cerrada y con escala precisa en niveles. Los valores más elevados se concentran en cómo los dispositivos los han motivado y ayudado en la comprensión. 
Gráfico 2. Porcentaje de respuesta del alumnado sobre el grado en el que las actividades planteadas les han ayudado a la mejora de su competencia comunicativa

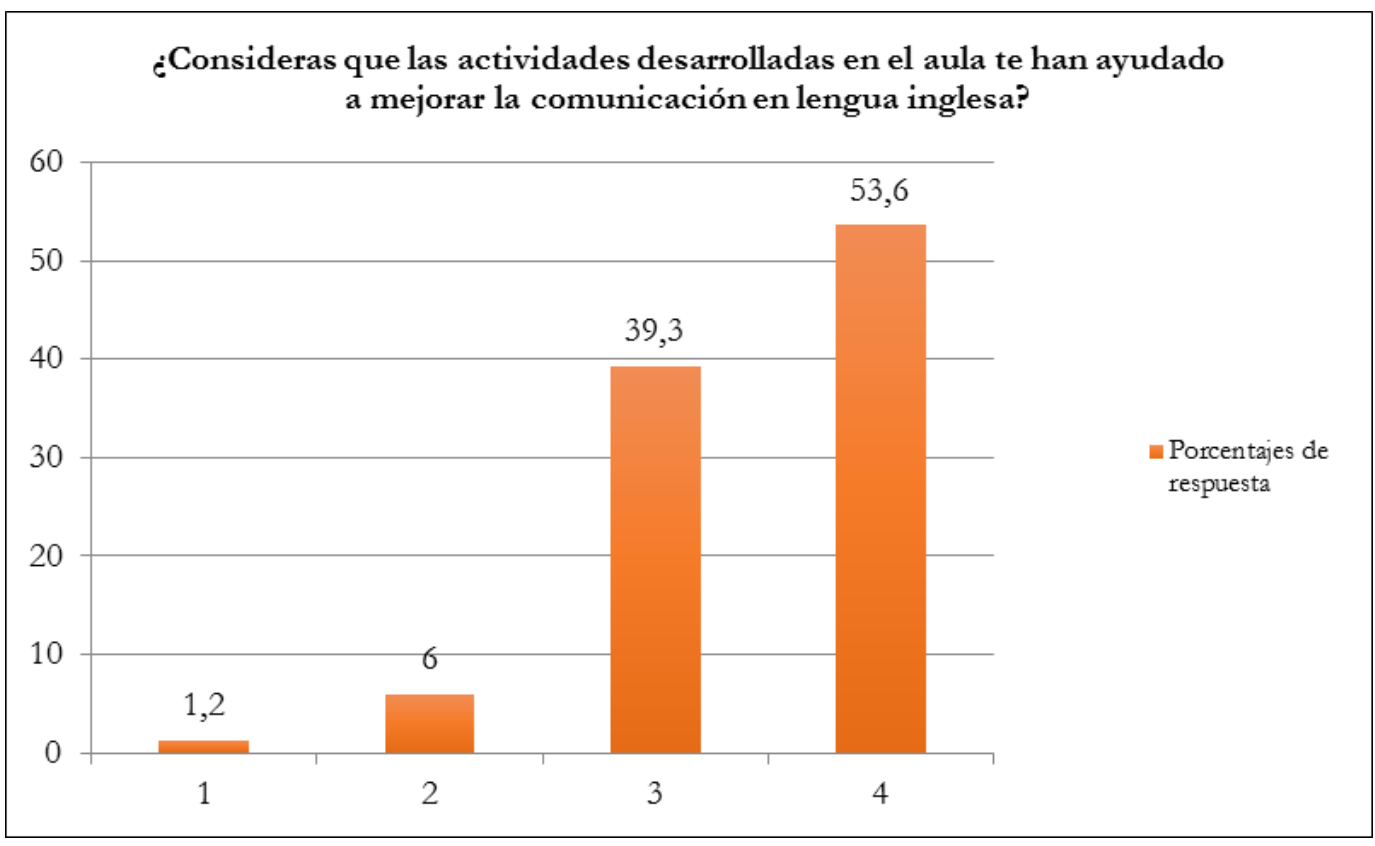

Fuente: elaboración propia.

En relación a la percepción que el alumnado tiene sobre su competencia tecnológica, los datos muestran que el proyecto ha supuesto que el alumnado utilice nuevas aplicaciones, ya que el 79,8 \% señala haber conocido durante el proyecto nuevas aplicaciones móviles, mientras que el 20,2 \% dice que ya conocía las aplicaciones utilizadas, siendo la aplicación favorita de la mayoría Viva Vídeo.

En cuanto a la motivación, los resultados obtenidos ponen de relieve que la utilización de dispositivos móviles resulta divertida para aprender inglés y por ello inferimos que les motiva. Una gran mayoría opta por puntuaciones altas: un 76,2 \% opina que «mucho» y un 21,4 \% opina que «bastante», frente a un 2,4\% que opina que «poco» o «nada». Estas valoraciones revelan la relación directa que se desprende entre uso de tecnología móvil y aprendizaje del inglés. El Gráfico 3 que se ofrece responde a conocer el nivel de respuestas sin estimar las valoraciones que son las que revelan datos más significativos procedentes de ubicar valor en escala Likert. 
Gráfico 3. Porcentaje de respuestas sobre el grado en el que alumnado disfruta aprendiendo lengua inglesa utilizando en el uso de dispositivos móviles

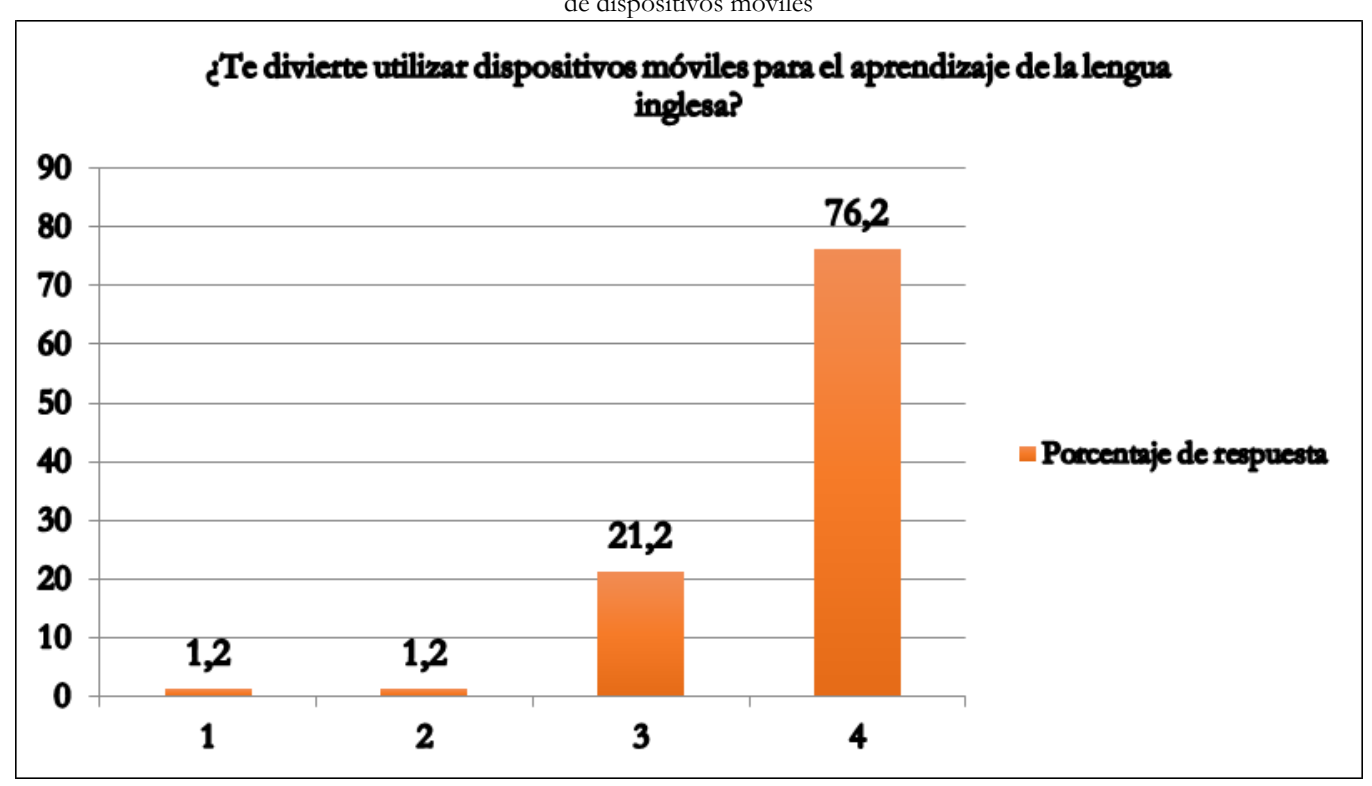

Fuente: elaboración propia.

\subsection{ENTREVISTAS AL PROFESORADO}

Las categorías utilizadas en la entrevista nos han permitido organizar y contrastar la información obtenida a través de la observación y los cuestionarios dirigidos al alumnado ofreciendo ideas para actuaciones de futuro. Para facilitar su compresión, los resultados se han organizado en función de las categorías utilizadas en la entrevista semi-estructurada. Se recogen inferencias de las opiniones y citas literales. Para garantizar el anonimato de las profesoras se utiliza una identificación codificada (P1, P2, etc.).

Las profesoras participantes en este proyecto consideran que las actividades llevadas a cabo han favorecido la mejora de la competencia comunicativa del alumnado en las lenguas del proyecto:

«La grabación de vídeos ha resultado una actividad muy divertida para los niños que se han esmerado mucho y se han esforzado por mejorar su pronunciación». P1

«Se han interesado en ver muchos tipos de vídeos en inglés antes de decidir lo que querían hacer y esta exposición a la lengua real les ha servido para mejorar su comprensión y su producción oral a la hora de hacer el vídeo». P3

Otra profesora opina que la necesidad imperante de hablar en inglés les fuerza y les motiva para utilizar la L2. Los alumnos quieren sorprender a todos aquellos que están a su alrededor: los profesores de prácticas y sobre todo a los auxiliares de conversación y también al resto de los compañeros y a sus padres. «Cuando existe necesidad comunicativa, ellos se esfuerzan». P4.

En cuanto a la competencia tecnológica P3 opina que el profesorado no está lo suficientemente formado y hay muchos otros profesores que piensan que el uso de móviles en el aula lo único que hace es distraer al alumnado. «Debemos aprender muchísimo más sobre esto porque es una motivación para el alumnado y los alumnos nacieron con esta tecnología». 
Todas las participantes están de acuerdo en las muchas ventajas que ofrece el aprendizaje con dispositivos móviles, pues los alumnos están acostumbrados a utilizarlos y conocen su funcionamiento mejor que los profesores. «Son un recurso accesible cuyo uso en las aulas motiva a los estudiantes fácilmente». P3. «Gracias a estos dispositivos el acceso a los recursos es muy rápido y la adecuación a las tareas es posible». P2. Además, las profesoras coinciden en destacar como una fortaleza la inmediatez para el registro de intervenciones de los alumnos: grabaciones audio, vídeo, etc. No obstante, se indican también algunas debilidades, como son los problemas técnicos de compatibilidad de las aplicaciones, fallos en las mismas o problemas de acceso a internet. La profesora P4 considera que el uso de la tecnología móvil en la escuela puede dar lugar a problemas cuando se hace un uso indebido de las mismas, por ejemplo, cuando el alumnado graba y difunde imágenes dentro del centro escolar ajenas a la actividad que se está realizando. En este sentido la profesora P1 considera que lo primero que hay que hacer es informar bien al alumnado sobre los problemas que el uso indebido de los dispositivos móviles puede acarrear, educarles en este sentido y firmar un tipo de contrato con el alumnado por el que se comprometan a cumplir un acuerdo sobre su uso en el centro educativo. En general, consideran que esta experiencia ha supuesto un aprendizaje en el campo de las TIC tanto para el alumnado como para el profesorado.

La profesora P4 considera que estas actividades ayudan a desarrollar la competencia artística del alumnado, pues estos se esfuerzan a la hora de crear y de presentar sus trabajos, ya que quieren que sus fotografías y sus videos gusten a los demás. P1 opina que esta actividad desarrolla la creatividad del alumnado y que trabaja la competencia artística, pues los alumnos han de diseñar sus montajes y argumentos y han de decidir qué aplicaciones utilizar para crear sus productos. Es decir, los alumnos se esfuerzan en ser originales y crear historias, personajes o escenarios diferentes y las aplicaciones móviles les ayudan mucho en este sentido por las múltiples posibilidades que tienen.

Las cuatro docentes coinciden en que la cooperación entre ellas ha sido excelente y el intercambio de experiencias y la comunicación ha sido fluida y eficaz.

La profesora $\mathrm{P} 3$ no tenía conocimientos previos sobre $m$-learning antes de iniciar el proyecto, pero considera que la competencia digital es fundamental en su formación. La profesora P4 comparte la misma opinión.

Todo el proceso de diseño e implementación de este proyecto ha sido muy motivador para las docentes: «me ha permitido experimentar con un recurso que no había utilizado antes en clase». P2. Además, todas ellas coinciden en que los alumnos se han sentido motivados en este proyecto, aunque se observaban diferencias. La motivación es otro de los aspectos más importantes a juzgar por lo recogido en estas entrevistas. Las profesoras coinciden en afirmar que durante la realización de las actividades con dispositivos móviles los alumnos se concentran en ello como si no hubiera nada más importante que hacer.

\subsection{RESULTADOS GENERALES}

Se puede decir que el proyecto 'Films \& Photographs: An M-learning Experience in Primary Education' obtuvo buenos resultados teniendo en cuenta que todos los participantes valoraron positivamente los aprendizajes. Esta valoración positiva se dio en relación a la comunicación lingüística y a la competencia tecnológica (uso de dispositivos móviles, smartphones y tablets). Además, permitió integrar nuevas técnicas de expresión artística y tecnológica tales como la producción y edición de fotografía y el montaje de películas, utilizando los dispositivos señalados. El alumnado realizó una enorme producción, tanto de fotografías como de vídeos, que se expusieron y se visionaron en el aula. Las fotografías, editadas con distintas aplicaciones móviles, sirvieron como 
recurso para el desarrollo de actividades orales: descripción, diálogo, narración, expresión de gustos y de sentimientos. Para la realización de los videos, los alumnos tuvieron que realizar algunas actividades lingüísticas previas tales como el diseño de los diálogos o de los textos descriptivos que iban a acompañar a estos vídeos. Pese a la dificultad de realizar estas tareas en lengua inglesa, los alumnos se esforzaron mucho y pusieron gran empeño en el desarrollo de las mismas. Otro aspecto a destacar es la libertad creativa con la que contaron para diseñar sus productos visuales y audiovisuales. El uso de estos recursos y las características del proyecto influyeron muy positivamente en la motivación del alumnado y del profesorado, lo cual repercutió también en el desempeño de las tareas de enseñanza y de aprendizaje. En el caso del profesorado, resultó una experiencia muy enriquecedora por la colaboración entre enseñantes de distintas etapas educativas (Educación Primaria y Educación Superior). El grado de implicación y cooperación fue muy alto y por ello todos opinan que el proyecto fue plenamente satisfactorio y muy motivador. En el caso del alumnado, resultó también una experiencia muy rica que les ayudó a trabajar colaborativamente en las actividades planteadas en el marco del proyecto. Por su parte, las profesoras opinaron que los alumnos, a través de sus prácticas de aula, mejoraron su capacidad para resolver problemas, aceptar las opiniones de sus compañeros y tomar decisiones consensuadas.

Cabe señalar que la implementación del proyecto no resultó un camino exento de dificultades, la primera estuvo relacionada con la escasa experiencia en la utilización de las aplicaciones sugeridas en el marco del proyecto, lo que provocó en ocasiones inquietud por parte del alumnado y el profesorado. La temática libre ocasionó también cierta intranquilidad entre el alumnado al inicio de la actividad, ya que estos estaban acostumbrados a una enseñanza más guiada Incluso el uso de los dispositivos móviles, por parte de los alumnos durante la clase, propició algún desencuentro con el equipo directivo del centro. Finalmente se solucionó autorizando las grabaciones de vídeo solo con los dispositivos del profesorado, con las implicaciones que esta decisión tuvo para el desarrollo de la experiencia.

\section{DiSCUSIÓN}

Los resultados obtenidos en esta investigación-acción dan cuenta de lo positivo que tiene el uso de las fotografías y los videos para fomentar la comunicación oral. Los alumnos pueden describir las fotografías, narrar qué hechos llevan aparejadas, establecer un diálogo sobre ellas, etc. No se puede olvidar que estas llevan asociada una vivencia, lo cual es muy importante para dotar de contenido la comunicación y estimularla. Tras cada presentación, los compañeros intervienen preguntando, opinando, y entre todos se produce lo que Fisher (2009) denomina diálogo creativo. Fisher hace una interesante propuesta para destacar el trabajo en la escuela en torno a los usos orales en la narración, la interrogación, la conversación, la discusión y el diálogo. Este autor expone su teoría sobre el diálogo como clave para mejorar la educación de los niños y generar interacción oral entre estos y sus maestros y cuidadores. Los diálogos son creativos cuando son abiertos, permiten opiniones diferentes e incluyen puntos de vista críticos que estimulan ideas nuevas. Este aspecto es un pilar esencial del proyecto que aquí se presenta y que, en sus actividades, busca y genera este diálogo creativo a través de la presentación de las fotografías, por ejemplo. En este sentido, no podemos olvidar la obra de Burbules (1999), como investigador de referencia en este campo, que estudia la complejidad y espontaneidad del diálogo como forma de comunicación pedagógica.

Los resultados de la investigación-acción que aquí se presentan se suman a los de otros autores como Bustillo, Rivera, Guzmán y Ramos Acosta (2017) que opinan que el uso de las aplicaciones móviles para la enseñanza y aprendizaje de las lenguas se ha incrementado en los últimos años. 
Además, hay artículos y ensayos que muestran los beneficios de la incorporación del uso de las tecnologías inalámbricas tales como Ramos y Valderruten (2014) que ha aplicado esta tecnología en el diseño e implementación de test para el aula de lenguas extranjeras. Otro autor, González (2015) presenta una investigación en la que a través de un cuestionario demuestra que el uso de la tecnología móvil mejora la calidad de las clases de idiomas y promueve la motivación hacia el aprendizaje. Autores como Gómez y Lazo (2015) consideran que el uso de aplicaciones en los contextos educativos requiere un diseño metodológico que contemple la experimentación, la simulación y el juego como elementos facilitadores del aprendizaje. Bustillo et al. (2017) concluyen, al igual que los autores mencionados, que el aprendizaje móvil repercute, en el proceso de aprendizaje de una lengua extranjera, en beneficios positivos en el desarrollo de competencias y específicamente en la mejora de la comprensión auditiva, Jung (2015) propone que el papel de la tecnología móvil en la enseñanza de la lengua inglesa se ha incrementado considerablemente en las últimas décadas, pues permite una buena conjunción con los objetivos de aprendizaje y su estudio, al igual que el que se presenta en este artículo, se centra en la perspectiva del estudiante y su percepción sobre la interacción de estos con esta tecnología. En cuanto a lo relacionado con la investigación-acción aquí presentada y coincidiendo con Oja y Pine (1981, pp. 9-10), los elementos básicos de la investigación cooperativa se resumen en que los problemas de investigación son mutuamente definidos por profesores e investigadoras; los docentes de la universidad y el profesorado de los centros educativos colaboran en la búsqueda de soluciones para los problemas detectados; los resultados de la investigación son utilizados en la solución de los problemas detectados; los docentes mejoran sus competencias de investigación en cuanto realizan el trabajo de campo y la autoría de los informes de investigación se realizan de manera conjunta.

\section{CONCLUSIONES}

Investigación y docentes debemos implicarnos, en colaboración, en la búsqueda de estrategias metodológicas y recursos dirigidos a la mejora de la competencia comunicativa del alumnado, pues la comunicación es uno de los pilares básicos del aprendizaje. La investigación-acción aquí presentada, sobre m-learning en el aula de lengua inglés es una muestra del interés por innovar utilizando nuevos recursos y metodologías centradas en el alumnado, dentro del contexto de la Educación Primaria. Se pone de relieve la importancia que tiene el uso de las nuevas tecnologías y en concreto de los dispositivos móviles, pues estos coinciden con los intereses y aficiones de niños y adolescentes. Aunar ambos presupuestos produce innovación para apoyar los conocimientos que se transmiten confluyendo en un proceso de aprendizaje significativo.

Ahora bien, que los niños y jóvenes manejen la tecnología no significa que sepan hacer un uso responsable de las misma, por lo que entre los objetivos formativos hay que contemplar tanto conocimientos o saberes, destrezas y habilidades o saber hacer (por ejemplo, el uso de las tecnologías), como la competencia existencial (saber ser, actitudes, motivaciones, valores, etc.). Por todo ello, los docentes deben ser conscientes de las fuerzas y debilidades del uso de las tecnologías de la información en las prácticas educativas, y trabajar en colaboración con las familias, procurando una enseñanza efectiva y afectiva que tenga en cuenta los aspectos motivacionales, tan importantes en la enseñanza de lenguas. El m-learning facilita el acceso a un mundo de información, por ello es útil incorporarlo en las prácticas de enseñanza de las lenguas con conocimiento, y disfrutando de cuanto de positivo tiene. La escuela ha de adaptarse a la realidad de la sociedad actual y para ello debe incorporar las tecnologías de la información y la comunicación (TIC) y las tecnologías para el aprendizaje de la comunicación (TAC), pues no debemos olvidar que la tecnología móvil se ha 
convertido en el lápiz y el papel de nuestros días. Estos dispositivos, como todos sabemos, incluyen una cámara fotográfica, una cámara de video, una grabadora de audio, una calculadora, un reloj, un calendario y una conexión a internet, entre otras muchas aplicaciones que hacen de ellos dispositivos muy versátiles y manejables con infinitas posibilidades didácticas. Por este motivo, los centros educativos deberían incorporar su uso a la mayor brevedad posible. La escuela no puede tener alumnos del siglo XXI, profesores del siglo XX y recursos y metodologías del siglo XIX (Acaso, 2013).

A modo de cierre, destacamos la importancia de la educomunicación por ser un concepto que integra la competencia comunicativa en el entorno escolar y que es un aspecto clave en nuestro proyecto. El m-learning, a su vez, es el otro concepto decisivo del proyecto que tiene un efecto muy motivador en el alumnado y contribuye a formar y educar ciudadanos bien informados, críticos, responsables y solidarios.

\section{BIBLIOGRAFÍA}

Acaso, M. (2013). Reduvolution, hacer la revolución en la educación. Barcelona: Espasa libros.

Alsina, M., Cañabate, D. y de la Creu, J. (2013). Competencias comunicativas para la acción didáctica. En R. Juanola y R. Rickenmann (eds.) La acción didáctica conjunta (pp. 171-182). Girona: Documenta Universitaria.

Ballesteros, C. y Palou, J. (2005). Las creencias del profesorado y la enseñanza de la lengua oral. En M. Vilá (coord.), El discurso oral formal (pp. 101-116). Barcelona: Graó.

Barbas, A. (2012). Educomunicación: Desarrollo, enfoques y desafíos en un mundo interconectado. Foro de Educación, 14, 155-175.

Bonnet, G. (ed.). (2002). The assessment of pupils' skills in English in eight European Countries. Disponible en: < http://www.mecd.gob.es/dctm/ievaluacion/internacional/habilidadesingles2002.pdf?docume ntId=0901e72b8011054e $>$. (Consultado el 23 de diciembre de 2017).

Burbules, N. (1999). El diálogo de la enseñanza. Buenos Aires: Amorrortu.

Bustillo, J., Rivera, C., Guzmán, J. y Ramos Acosta, L. (2017). Benefits of using a mobile application in learning a foreign language. Sistemas \& Telemática, 15(40), 55-68.

Cantillo, C., Roura, M. y Sánchez, A. (2012). Tendencias actuales en el uso de dispositivos móviles en educación. La educación digital magazine, 147. Recuperado de < http://www.educoas.org/portal/la educacion digital/147/pdf/ART UNNED EN.pdf>.

(Consultado el 22 de diciembre de 2017).

Carr, W. \& Kemmis, S. (1988). Teoría crítica de la enseñanza. La investigación-acción en la formación del profesorado. Barcelona: Martínez-Roca.

Consejo de Europa. (2002). Marco Común Europeo de Referencia para las lenguas: aprendizaje, enseñanza, evaluación. Madrid: MECD, Instituto Cervantes y Ed. Anaya.

Council of Europe (2001). Common European Framework of Reference for Languages: Learning, teaching, assessment. Cambridge: Cambridge University Press.

Dörnyei, Z. (2001). Teaching and researching motivation. Harlow: Longman.

Dörnyei, Z. (2002). The motivational basis of language learning tasks. In P. Robinson (ed.), Individual differences and instructed language learning (pp. 137-158). Amsterdam: John Benjamins.

Dörnyei, Z. (2005). The psychology of the language learner: Individual differences in second language acquisition. Mahwah (NJ): Lawrence Erlbaum.

Elliot, J. (1990). La investigación-acción en educación. Madrid: Morata. 
España: Ley Orgánica 2/2006, de 3 de mayo, de Educación (LOE). Boletín Oficial del Estado no 106 de 4 de mayo.

España: Ley Orgánica 8/2013, de 9 de diciembre, para la mejora de la calidad educativa (LOMCE). Boletín Oficial del Estado no 295 de 10 de diciembre.

Fisher, R. (2009). Creative Dialogue: Talk for thinking in the classroom. London: Routledge.

Fombona, J. y Pascual, M. A. (2013). Beneficios del m-learning en la educación superior. Educatio Siglo XXI, 31(2), 211-234.

Gardner, H. y Davies, K. (2014). La generación APP. Cómo los jóvenes gestionan su identidad, su privacidad y su imaginación en el mundo digital. Barcelona: Paidós.

Goh, C. y Burns, A. (2012). Teaching Speaking. Cambridge: Cambridge University Press.

Gómez, C. y Lazo, C. (2015). Modelo de integración educomunicativa de apps móviles para la enseñanza y aprendizaje. Revista de Medios y Educación, 46, 137-153.

González, H. (2015). La integración de la tecnología como herramienta significativa en la enseñanza de inglés como lengua extranjera. Revista Horizontes Pedagógicos, 17, 53-66.

Hughes, R. (2015). Teaching and researching: Speaking. (2nd Ed.). New York (NY): Routledge.

Hymes, D. (1972). On communicative competence. In J. B. Pride and J. Holms (eds.), Sociolinguistics (pp. 269-293). Harmondsworth: Penguin.

Ibarrola, B. (2013). Aprendizaje emocionante. Neurociencia para el aula. Madrid: SM.

Jung, H. (2015). Fostering an English teaching environment: Factors influencing English as a foreign language teachers' adoption of mobile learning. Informatics in Education, 14(2), 219-241.

Kaplún, M. (1998). Una pedagogía de la comunicación. Madrid: De la Torre.

Lewin, K. (1946). Action research and minority problems. Journal of Social Issues, 2(4), 34-46.

Levy, M. (2012). Technology in the classroom. In A. Burns, J. C. Richards (eds.), The Cambridge guide to pedagogy and practice in second language acquisition (pp. 279-286). Cambridge: Cambridge University Press.

López, N. y Gómez, L. (2016). Social Mobile: Los medios a través de la cuarta pantalla. Fonseca, Journal of Communication, 12, 7-9.

López, F. y Silva, M. (2013). Factores que inciden en la aceptación de los dispositivos móviles para el aprendizaje en educación superior. Estudios sobre Educación, 30, 175-195.

Martínez-Salanova, E. (2016). Educomunicación. Recuperado de $<$ http://www.uhu.es/cine.educacion/didactica/0016educomunicacion.htm $>$. (Consultado el 20 de diciembre de 2017).

McKerman, J. (1993). Varieties of curriculum action research: Constraints and typologies in American, British and Irish projects. Journal of Curriculum studies, 25, 445-458.

Ministerio de Educación, Cultura y Deportes. Estudio Europeo de Competencia Lingüística EECL. Volumen I. Informe español. Recuperado de <http://www.mecd.gob.es/dctm/ievaluacion/internacional/eeclvolumeni.pdf?documentId=09 01e72b813ac515>. (Consultado el 20 de diciembre de 2017).

Ramos, L. y Valderruten, A. (2014). Beneficios del uso de tecnologías inalámbricas de respuesta en exámenes de inglés. Contextos, 3(12), 45-54.

Reason, P. (1994). Participation in Human Inquiry. London: Sage Publications.

Rodríguez, G., Gil, J. y García, E. (1996). Metodología de la investigación cualitativa. Granada: Ediciones Aljibe.

Schumann, J. (1994). Where is cognition? Emotion and cognition in second language learning. Studies in second language acquisition, 16, 231-242. 
Scovel, T. (2000). Learning New Languages: A Guide to Second Language Acquisition. Boston (MA): Heinle \& Heinle.

Soto, E y Pérez Gómez, A. (2015). Lessons Studies: un viaje de ida y vuelta recreando el aprendizaje comprensivo. Revista Interuniversitaria de Formación del Profesorado, 29(3), 15-28.

Stenhouse, L. (1975). An Introduction to Curriculum Research and Development. London: Heinemann Educational Books.

Stenhouse, L. (1998). Investigación y desarrollo del currículum. Madrid: Morata.

Ushioda, E. (2012). Motivation. In A. Burns \& J. C. Richards (eds.), The Cambridge guide to pedagogy and practice in second language acquisition (pp.77-85). Cambridge: Cambridge University Press.

Vilà, M. y Castellá J. M. (2014). 10 ideas clave. Enseñar la competencia oral en la clase: aprender a hablar en público. Barcelona: Graó.

Vilà, M. y Castellá, J. M. (2015). La lengua oral formal: características lingüísticodiscursivas y reflexiones sobre su enseñanza. En J. Mata, P. Núñez y J. Rienda (coords. y eds.), Didáctica de la lengua y la literatura (pp. 55-63). Madrid: Pirámide.

Viñals-Blanco, A. (2016). El Ocio Conectado, móvil, transmedia y multisoporte de los jóvenes en la Era Digital. Fonseca, Journal of Communication, 3, 99-113. 